\title{
Emission features in a B[e] binary system V2028 Cyg
}

\author{
Jan Polster ${ }^{1,2}$, Daniela Korčáková ${ }^{2}$, Viktor Votruba ${ }^{1,2}$, Petr Škoda $^{2}$, \\ Miroslav Šlechta ${ }^{2}$ and Blanka Kučerová ${ }^{1}$ \\ ${ }^{1}$ Faculty of Science, Masaryk University in Brno, Kotlářská 2, 61137 Brno, Czech Republic \\ ${ }^{2}$ Astronomical Institute AV ČR, Fričova 298, 25165 Ondřejov, Czech Republic \\ email: polster@physics.muni.cz, kor@sunstel.asu.cas.cz
}

\begin{abstract}
We present a preliminary analysis of our six-year observation campaign of the $\mathrm{B}[\mathrm{e}]$ stellar system V2028 Cyg (MWC 623). The time variability of spectral features is described.
\end{abstract}

Keywords. stars: emission-line, binaries: general, stars: individual (V2028 Cyg)

\section{Introduction}

A variable star V2028 Cyg (MWC 623) shows a B[e] phenomenon. The nature of the central object is very difficult to determine due to the extended circumstellar medium. The spectrum of V2028 Cyg is dominated by strong Balmer emissions, especially $\mathrm{H} \alpha$. It also contains permitted and forbidden emission lines of low-ionized or neutral atoms (Fe II, [Fe II], [O I], ...).

We present measurements of some emission features in spectra of our six-year observation campaign.

84 spectra in the $\mathrm{H} \alpha$ region were taken with the Ondřejov $2 \mathrm{~m}$ telescope using a coudé slit spectrograph with resolving power $R_{\mathrm{H} \alpha} \sim 12500$. Additional four spectra were obtained from the ELODIE archive $(R=42000$ in the wavelength interval $3906-6811$ $\AA)$.

\section{Radial velocities and bisectors}

Radial velocities of the metallic emissions were measured by Gaussian fitting. We used the double Gaussian method (Schneider \& Young, 1980) for the wings and the polynomial fitting in the case of the peak to measure the $\mathrm{H} \alpha$ radial velocities. The overall behaviour of the $\mathrm{H} \alpha$ line profile is described by bisectors (Fig. 2).

\section{Conclusions}

Radial velocities of the metallic emissions (Fig. 1) show certain scatter, but no period or trend was found. There is a clear minimum of radial velocity in JD $=2454155 \pm 17$ in the case of $\mathrm{H} \alpha$ wings (Fig. 3). The peak velocity values are more scattered, but there can be resolved a minimum in $\mathrm{JD}=2454683 \pm 33$.

The bisector measurements (Fig. 2) reveal, that the whole lower part of the H $\alpha$ profile (under the hump) behaves the same way as the wings. The peak above the hump varies differently. It indicates existence of at least two different kinematical environments in the envelope, where $\mathrm{H} \alpha$ emission arises. 

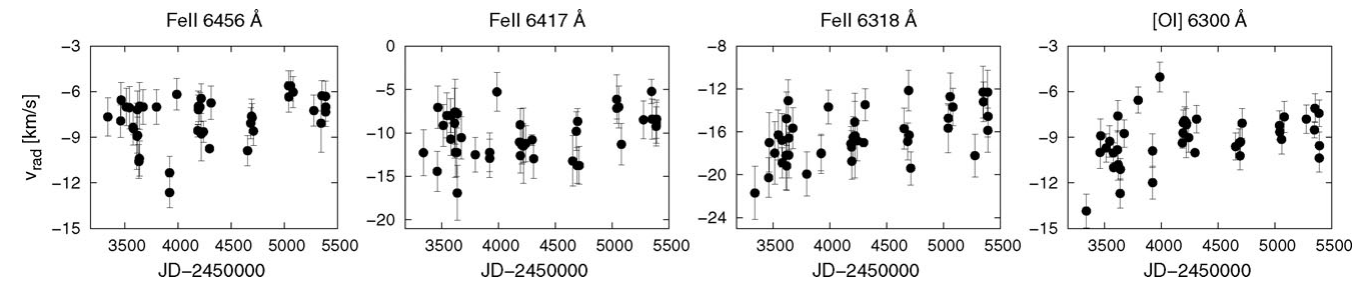

Figure 1. Radial velocities of [O I] and Fe II emission lines.
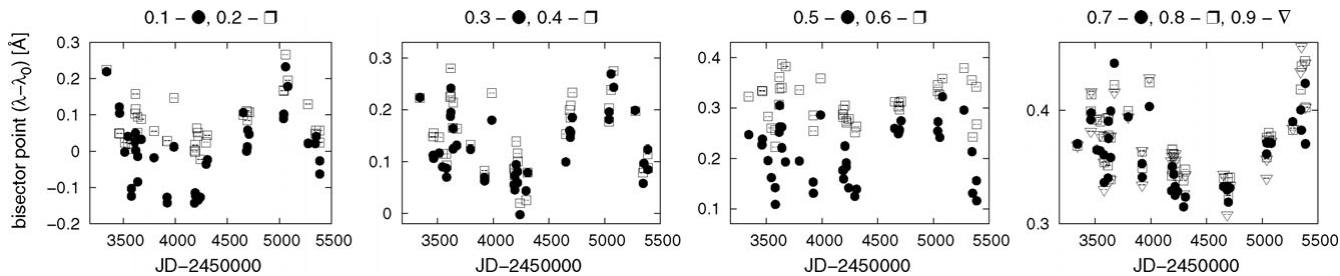

Figure 2. Bisector points of $\mathrm{H} \alpha$ line in relative heights from 0.1 (wings) to 0.9 (peak).
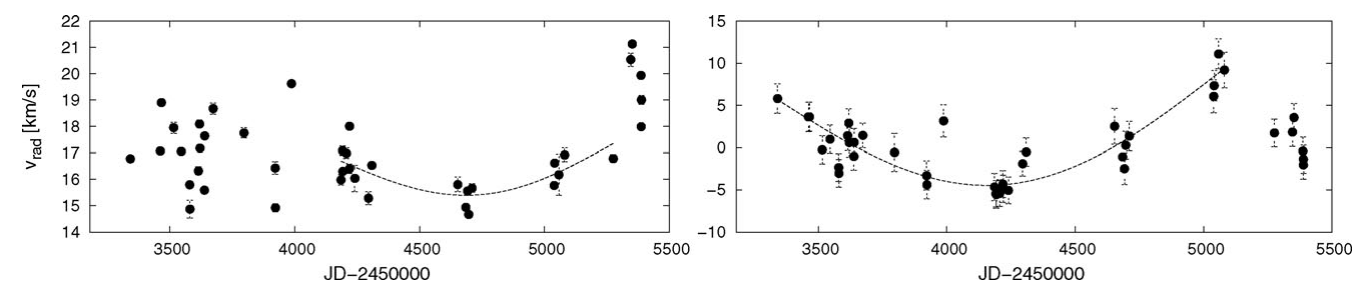

Figure 3. Radial velocities of the $\mathrm{H} \alpha$ peak (left) and wings (right). The fitting function is plotted in the graph.

The half maximum widths of metallic lines are $\sim 1 \AA$, which restricts the maximum value of the velocity gradient in the region of their formation to $\sim 50 \mathrm{~km} / \mathrm{s}$. No observed line shows P Cyg profile and the emissions of metals have a blue-shifted maximum.

Currently, we are working on a numerical modeling of the bisector time variability in order to explain these observed phenomena.

\section{Acknowledgements}

This research is supported by grants 205/09/P476 (GA ČR), 205/08/H005(GA ČR) LC06041 (MŠMT ČR) and MUNI/A/0968/2009. The Astronomical Institute Ondřejov is supported by a project AV0Z10030501.

\section{Reference}

Schneider, D. P. \& Young, P. 1980, ApJ, 238, 946 